\title{
La radioprotection dans les centrales nucléaires d'Electricité de France ${ }^{\star}$
}

\author{
J. LECLERCQ** \\ (Manuscrit reçu le 20 mai 1985)
}

RÉSUME

Cet exposé traite plus particulièrement des méthodes et des résultats relatifs à la radioprotection dans les centrales nucléaires d'Electricité de France.

\section{ABSTRACT}

The methods and results concerning radiation protection in nuclear power plants of Electricité de France are presented and discussed.

\section{RESULTATS DE DOSIMETRIE DES CENTRALES FRANCAISES}

\subsection{L'exposition collective}

La dose annuelle moyenne par tranche a été de 208 h.rem pour 1984 ; elle est restée inférieure à 250 h.rem au cours des trois dernières années (fig. 1), avec des situations assez différentes par tranche ou ensemble de tranches. Rapportée à la production d'électricité, l'exposition collective est restée comprise entre 0,03 et $0,04 \mathrm{rem} / \mathrm{GWh}$ sur la période 19801984 (fig. 2).

On voit par ailleurs (fig. 3) l'évolution de la dose cumulée en fonction de l'énergie produite. On constate que la dose totale ramenée à l'énergie fournie, pour une centrale donnée, apparaît comme relativement invariante ; on note, également, une diminution notable de la dose cumulée en fonction de l'expérience acquise depuis Chooz jusqu'aux centrales de Gravelines, Dampierre et Tricastin.

Si la relative invariance dans le temps, que nous n'avons pas encore analysée en détail, ne peut s'expliquer en première analyse que par le cumul d'effets contraires (d'un côté vieillissement à la hausse, de l'autre progrès dans l'exploitation à la baisse), il existe un certain nombre d'explications à l'amélioration sensible entre $\mathrm{Chooz}$ et les tranches les plus récentes du CP1.

* Communication présentée au colloque organisé à Bruxelles du 20 au 22 mars 1985 par les Sociétés belge et française de radioprotection sur la "Radioprotection du personnel des entreprises extérieures intervenant dans les installations nucléaires".

$\star \star$ Electricité de France, Service de la production thermique, 3, rue de Messine, 75008 Paris. 


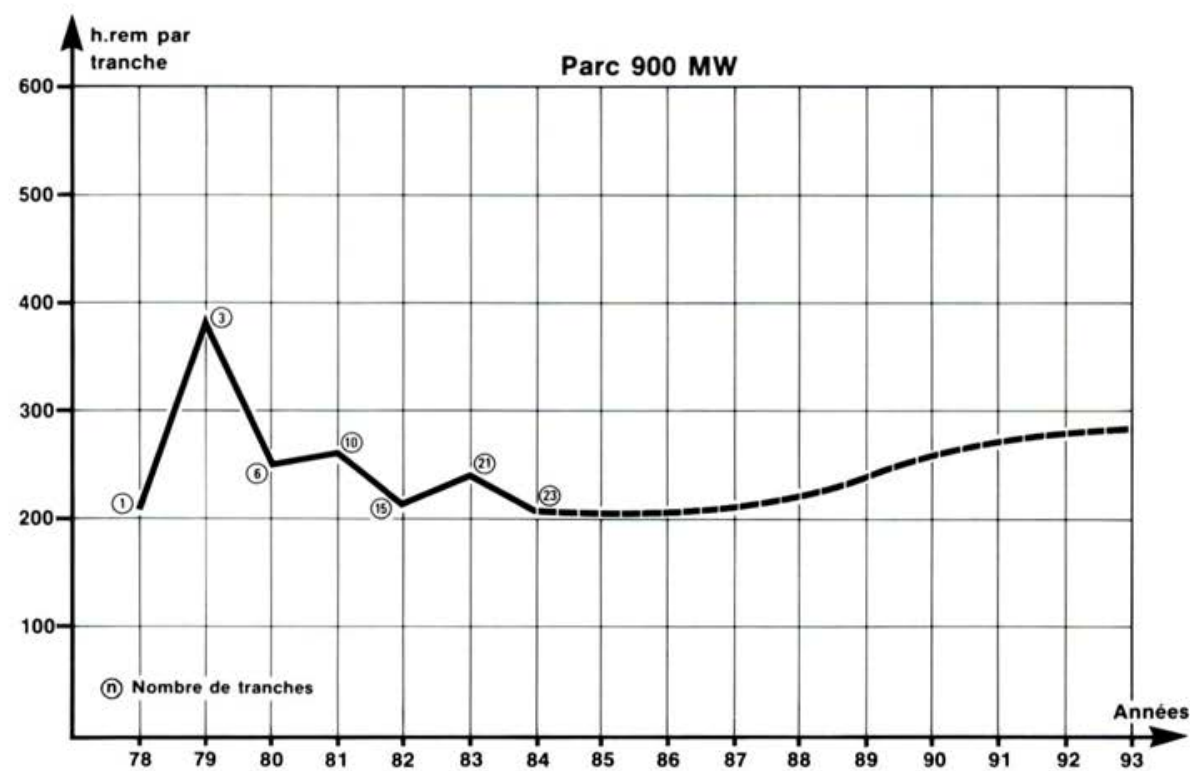

Fig. 1. - Dose collective annuelle par tranche ayant eu un arrêt dans l'année.

La centrale de Chooz, tranche unique qui, pour une même énergie produite, "vieillit" en fait quatre fois plus vite qu'un Centre de production nucléaire $(C P N)$ à 4 tranches, est un cas particulier, car elle accumule tous les facteurs allant dans le sens d'un accroissement des doses: pas d'effet palier, peu de retour d'expérience, effet de taille (seulement $300 \mathrm{MW}$ ), 4 boucles (donc plus de matériel), technologie certainement moins étudiée que le contrat programme de $900 \mathrm{MW}$, pas de dosimétrie électronique en temps réel (incitative à la réduction des doses), pas d'outillages spéciaux tels que ceux utilisés sur les tranches de $900 \mathrm{MW}$.

La centrale de Fessenheim, qui "vieillit" deux fois plus vite qu'un CPN à 4 tranches, ne dispose pas, non plus, de toutes les modifications qui ont été faites sur le CP.

Quant à ce qui différencie Bugey des centrales du premier contrat programme (Tricastin, Dampierre, Gravelines), il faut surtout insister sur l'effet bénéfique de l'homogénéité des tranches (formation du personnel, retour d'expérience, outillages adaptés, etc.).

\subsection{Quelques comparaisons internationales}

La figure 4 relative à la dose collective moyenne par réacteur et par an a été publié lors d'un congrès de l'Agence internationale de l'énergie 
atomique (AIEA). Elle présente les résultats de quelques pays pour les réacteurs à eau (LWR) et mérite quelques explications. Tout d'abord, nous manquons, à l'heure actuelle, d'informations précises sur certains pays. De plus, il faut pouvoir distinguer et expliquer les résultats entre PWR et $B W R^{(1)}$.

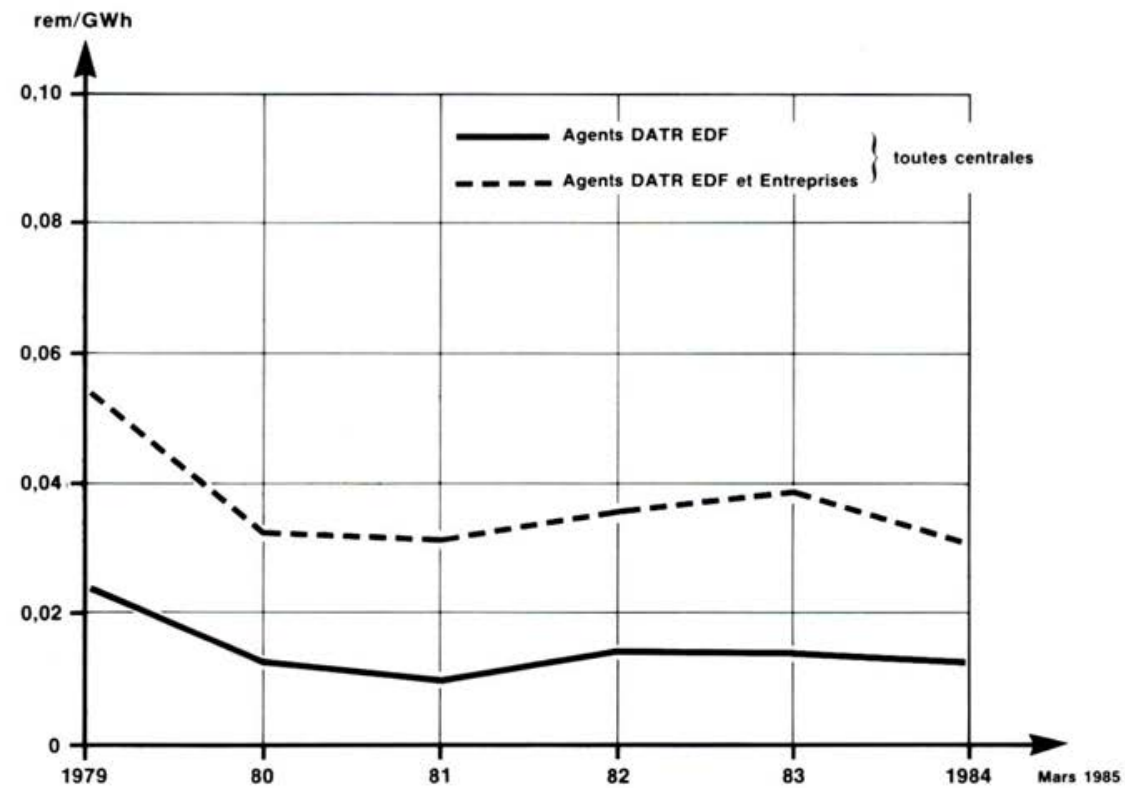

Fig. 2. - Doses collectives rapportées à la production.

En première analyse, nous savons que les résultats officiels des USA et du Japon sont largement supérieurs à la moyenne nationale alors que les résultats suédois par exemple, en provenance d'ASEA Atom, sont significativement peu élevés. Ceci dit, pour les PWR, les résultats japonais sont comparables aux français pour 1984.

II faut donc, avant de comparer des données internationales, être très prudent sur l'origine des données et obtenir des informations complémentaires, notamment sur les méthodes de comptabilisation des doses (avec ou sans seuil, automatique ou manuelle...). Ce travail est entrepris et permettra prochainement de présenter l'ensemble des valeurs relatives aux diverses exploitations.

(1) PWR ou REP : Pressurized water reactor, réacteur à eau sous pression ; BWR ou REB : Boiling water reactor, réacteur à eau bouillante. 


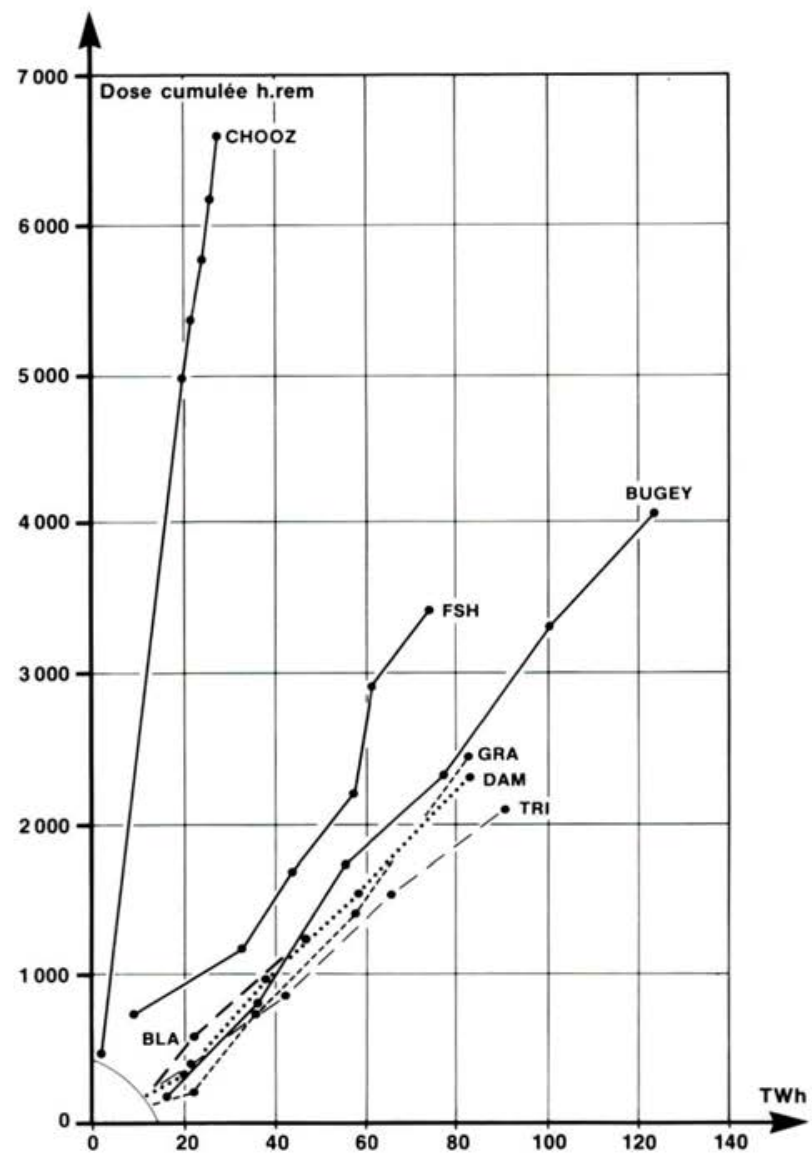

Fig. 3. - Comparaison des doses cumulées : centrales REP.

\subsection{Les doses individuelles}

En ce qui concerne la dosimétrie individuelle, rappelons que la DMA (dose maximale admissible) est de $5 \mathrm{rem} / \mathrm{an}$ et que la loi française définit clairement le partage des responsabilités entre l'employeur qui met son personnel à la disposition d'EdF et le chef de centrale qui fournit tous les éléments à l'entreprise pour qu'elle puisse exercer sa responsabilité.

La surveillance dosimétrique est assurée d'une part par un film réglementaire, dont le développement est centralisé par le Service central de 
protection contre les rayonnements ionisants (SCPRI), et par un dosimètre électronique, à lecture directe, non imposé par la législation, pris en charge par EdF. C'est pourquoi les valeurs présentées concernent principalement EdF. Sur la courbe de distribution des doses annuelles (fig. 5), on remarque (pour un effectif de 11000 agents DATR environ) que la moyenne est de 182 mrem et que la plus grande partie des agents a une dose annuelle autour de $18 \mathrm{mrem}$. Par ailleurs, il n'y a pas eu de dépassement de dose (5 rem/an ou 3 rem/trimestre) en 1984 (fig. 6).

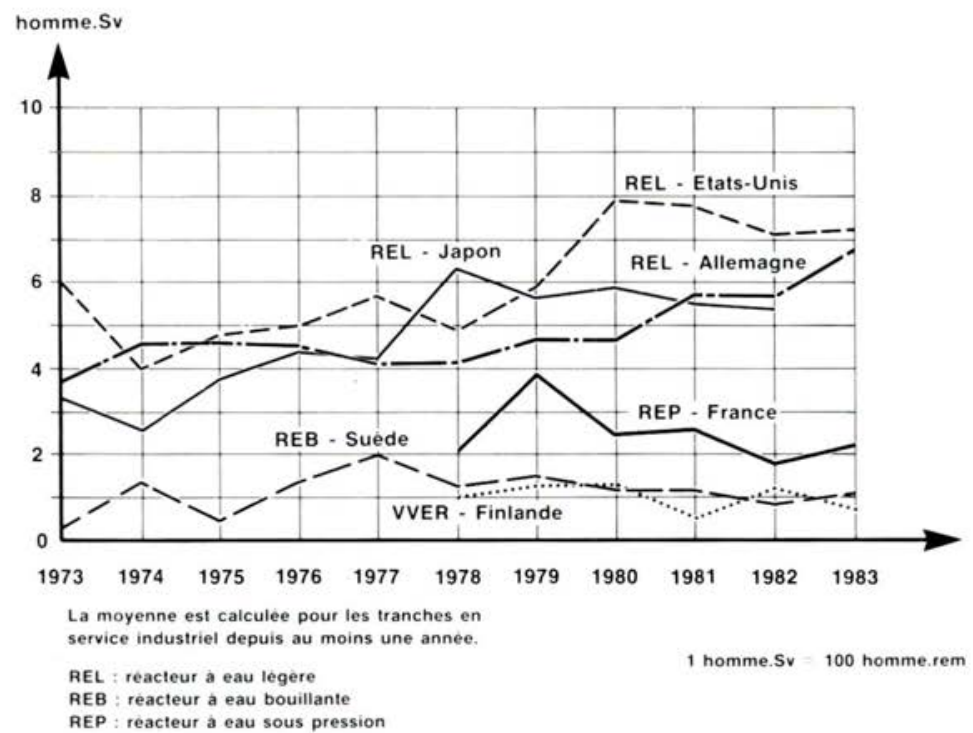

Fig. 4. - Dose collective moyenne, par tranche et par an.

Nous n'avons pas, à l'heure actuelle, la possibilité de faire ces analyses sur la dosimétrie entreprise et l'on signalera seulement, pour 1984, le cas de dépassement de la DMA, par contamination, pour un agent d'entreprise à Tricastin. Cependant, nous envisageons d'utiliser plus largement la dosimétrie électronique pour l'ensemble des agents sur un site donné, afin de comparer plus précisément les deux populations, et d'en tirer des enseignements pour les arrêts futurs.

\section{ORIGINE DES DOSES}

On examinera la répartition des doses selon les périodes d'activité, l'origine physique, les opérations et les agents concernés. 
- Arrêt et hors arrêt : la dosimétrie annuelle, en h.rem/réacteur.an, se répartit environ pour $25 \%$ hors arrêt et $75 \%$ en arrêt de tranche, pour une année comportant une visite normale. Dans les doses hors arrêt, il faut comprendre toutes les doses prises lors de l'exploitation globale de la centrale, ainsi que l'entretien qui s'effectue en fonctionnement à l'intérieur de lîlot nucléaire. Les doses d'arrêt totalisent les doses des agents EdF et des entreprises prestataires, et sont délivrées dans leur quasitotalité pendant les arrêts en fin de cycle pour rechargement.

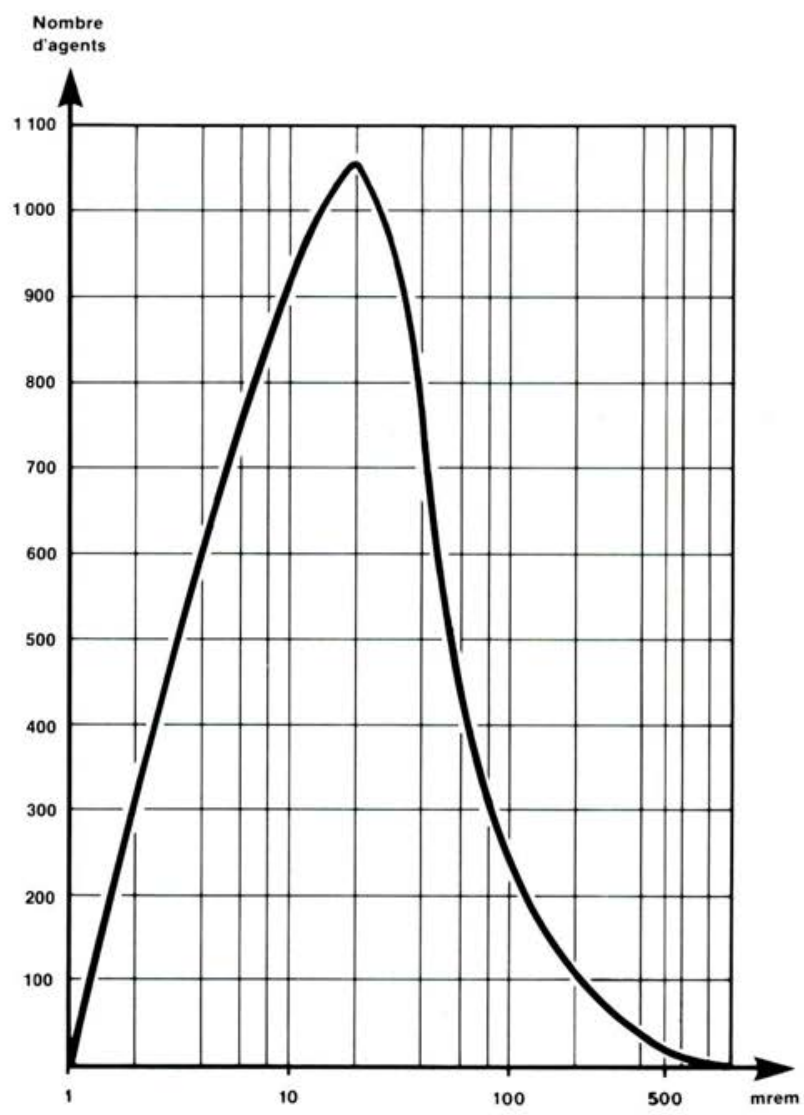

Fig. 5. - Répartition des doses délivrées en 1984 aux agents EdF (moyenne: $182 \mathrm{mrem} /$ agent EdF). 


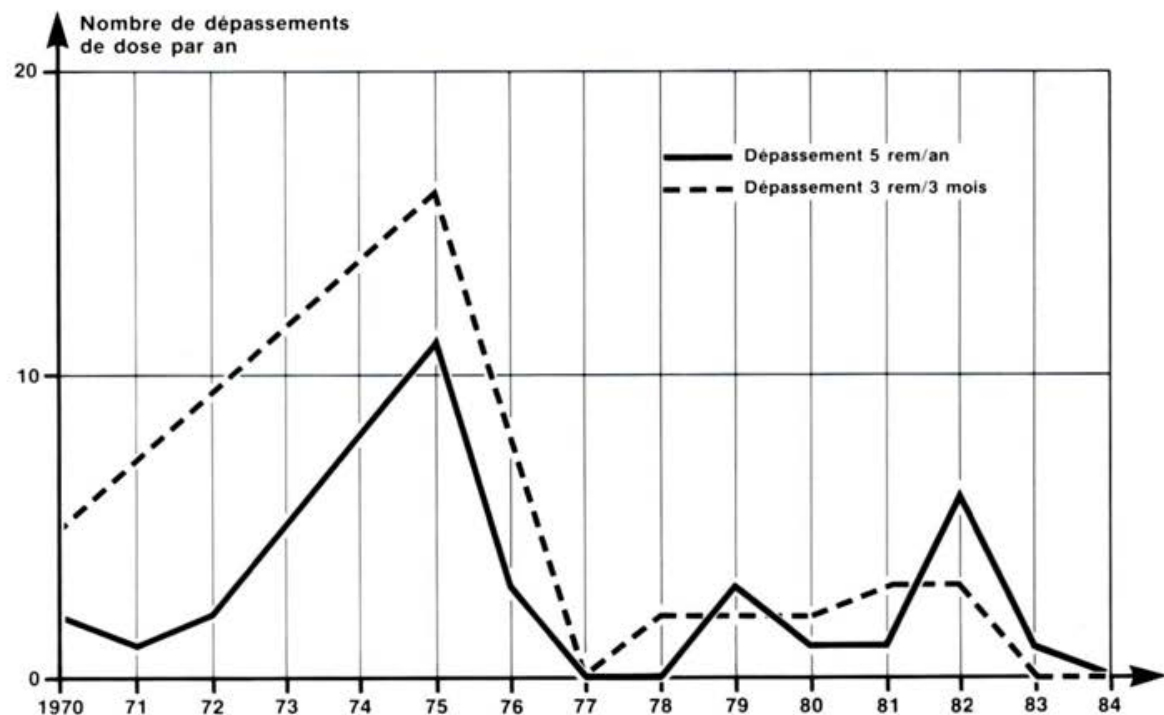

Fig. 6. - Dépassements de dose des agents EdF.

- Produits de fission et de corrosion : la répartition est la suivante :

. produits de fission

azote 16 et neutrons

structures activités

produits de corrosion
$5 \%$;

$5 \%$;

$5 \%$;

$85 \%$.

La part ci-dessus due aux produits de fission est estimée pour des cycles comportant des ruptures de gaines importantes, comme ce fut le cas à Bugey 2 et Tihange (uranium libre sous flux). Dans la majorité des cas, elle est négligeable.

Les produits de corrosion principaux sont le cobalt 60 (qui provient principalement des impuretés en cobalt 59 ) responsable de $60 \%$ de la dose totale, le cobalt 58 (qui provient du nickel 58) responsable de $15 \%$ de la dose totale, les divers autres produits tels que manganèse 54 , chrome 51 , fer 59 , responsables d'environ $10 \%$ de la dose totale.

La conclusion évidente de ces répartitions par origine est que la plus grande partie des doses délivrées aux travailleurs est due aux produits de corrosion déposés sur les circuits lors des interventions en arrêt de tranche, ce qui situe déjà les directions dans lesquelles nos efforts doivent être entrepris.

- Répartition par opération : bien qu'entachée d'une certaine dispersion, la dose se répartit relativement de la même façon, que la visite soit 
normale ou complète, sauf pour la partie relative aux inspections, plus importante dans le cas d'une visite complète (fig. 7 ).

VISITE NORMALE

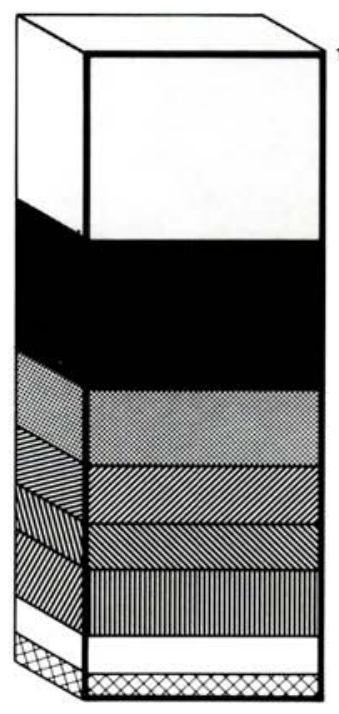

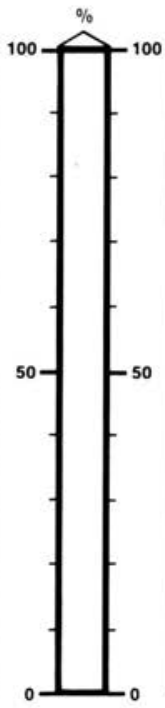

VISITE COMPLETE

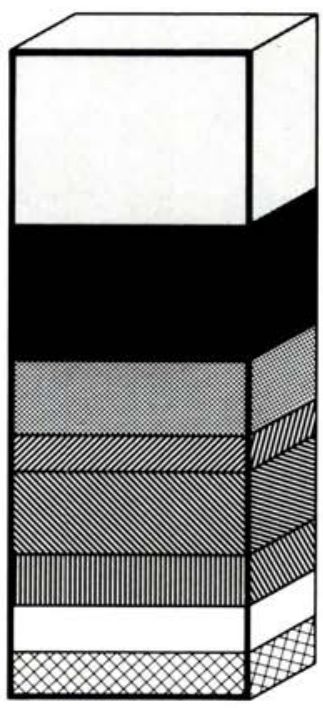

Servitudes (nettoyage, décontamination, preparation chantier)

Génèrateur de vapeur Ouverture, termeture cuve $\mathbb{Z}$ Circuit primaire

$\mathbb{N}$ Circuits RCV-RRA

III Autres circuits

Instrumentation coeur

$\square$ Manutention combustible

Inspections (hors GV)

Fig. 7. - Répartition des doses lors d'un arrêt pour rechargement.

Les opérations les plus importantes sont (en \% de la dose totale) :

. la préparation des chantiers

- les générateurs de vapeur

. l'ouverture de cuve (et la fermeture)
$30 \%$

$20 \%$

$10 \%$.

La dosimétrie prise en compte est en quasi-totalité due à une irradiation par les rayonnements gamma, car il est très facile de se protéger du rayonnement bêta, et les particules alpha ont une influence négligeable en l'absence de rupture de gaine importante. D'autre part, la dosimétrie neutron est de l'ordre de $1 \%$ de la dosimétrie globale.

- Agents d'EdF et entreprises extérieures : en comparant les résultats bruts de la dosimétrie annuelle, on constate que la dose accumulée par les agents d'entreprises extérieures représente 70 à $75 \%$ de la dose prise en arrêt de tranche (fig. 8) qui reprend la répartition pour les deux dernières années). Dans la mesure où les agents d'entreprises ne travaillent pratiquement pas hors arrêt de tranche, la répartition collective des doses entre agents EdF ou d'entreprises est du même ordre $(0,75$ $\times 0,75 \cong 0,50$ ). 


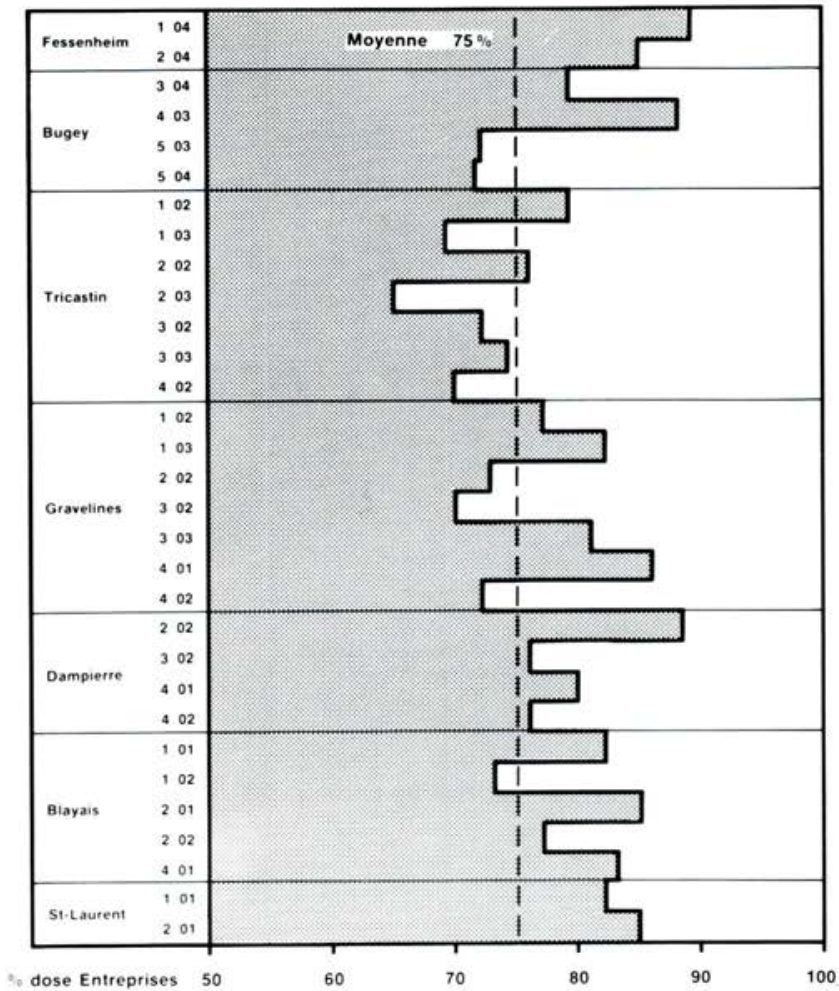

Fig. 8. - Dosimétrie d'arrêt relative des agents EdF et entreprises prestataires pour $1983 / 1984$.

Il faut également, si l'on veut comparer ces doses pendant les périodes d'arrêt, considérer le total des heures travaillées qui sont environ trois fois plus importantes pour les entréprises. A titre d'exemple, on peut observer la répartition des doses plus détaillées sur 2 arrêts de Blayais (fig. 9) entre agents d'entreprises et EdF. La dose totale est bien trois fois supérieure, mais rapportée à l'agent, l'écart n'est plus que de $50 \%$, le maximum dans les deux cas restant inférieur à 1,3 rem.

On se rappellera que, pendant les arrêts de tranche, les travaux de mécanique et de chaudronnerie sont pris en charge par des agents EdF à hauteur d'environ $35 \%$, le solde étant effectué par des entreprises à la fois pour assurer un retour d'expérience vers les constructeurs de matériel, également pour ne pas être conduit à un sous-emploi des équipes EdF hors arrêt de tranche, c'est-à-dire plus de six par an pour un site à quatre tranches. 


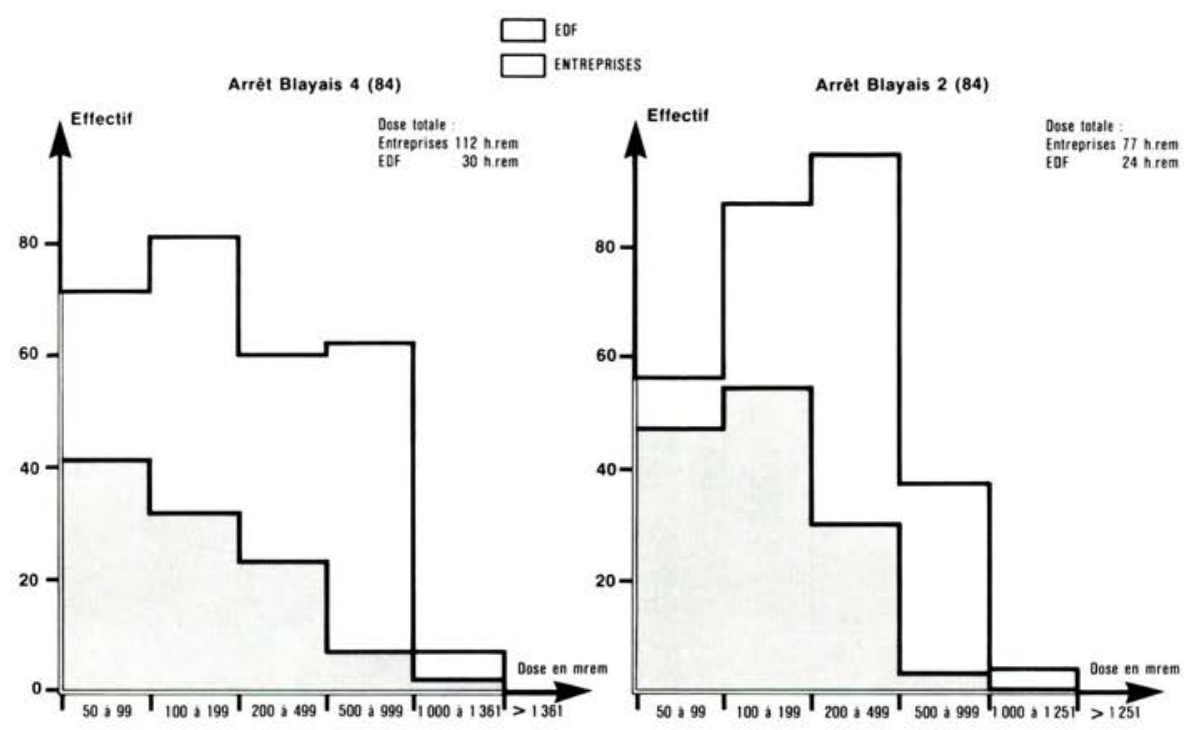

Fig. 9. - Distribution relative des doses - Entreprises/EdF.

\section{COMMENT REDUIRE LES DOSES ?}

L'énumération suivante ne vise pas à l'exhaustivité, mais montre, sur un certain nombre de points particuliers parmi les plus importants, la méthode suivie pour améliorer les résultats dans le domaine de la radioprotection.

\section{- Chimie du circuit primaire}

La plus grande partie des débits de doses étant provoquée par les produits de corrosion déposés sur les parois des circuits, nous avons cherché une chimie qui les minimiserait.

Les études théoriques menées sur ce sujet par le Commissariat à l'énergie atomique (CEA), à notre demande, nous ont montré, en prenant en compte les solubilités des espèces présentes dans le circuit, que :

- plus le $\mathrm{pH}$ est élevé et moins la probabilité de déposer sous flux est importante; on diminue donc l'activité spécifique ;

. plus le $\mathrm{pH}$ est bas et plus on minimise la masse de matière relâchée.

On a donc cherché l'optimum de ces deux exigences contradictoires. Celui-ci consiste à maintenir un $\mathrm{pH}$ constant et égal à 6,83 pendant une grande partie du cycle à l'aide du conditionnement à lithine décroissante. 
Pour des raisons de dépôts trop importants sur le combustible, on ne peut descendre en dessous de $0,7 \mathrm{ppm}$ de lithine, ce qui conduit à un $\mathrm{pH}$ légèrement croissant en fin de cycle. Les gains estimés par rapport à une chimie "libre" sont de $20 \%$ sur les débits de dose.

Pour l'avenir, sans remettre en cause le choix du principe du $\mathrm{pH}$ constant, il semble que la valeur absolue même de ce $\mathrm{pH}$ doive être légèrement augmentée, au vu des données traitées actuellement.

De plus, le conditionnement à lithine décroissante ne tient pas compte du strech-out qui se généralise sur l'ensemble du parc et de la fin du cycle, périodes pour lesquelles on fonctionne à $\mathrm{pH}$ variable (concentration en bore nulle, teneur en lithine constante et une température moyenne qui décroît pour récupérer la réactivité nécessaire au fonctionnement du réacteur).

Pour ces deux derniers points, une étude théorique est en cours, ainsi que des essais sur boucle, au CEA.

\section{- Procédure d'arrêt à froid}

La procédure d'arrêt consiste en une aération aussi rapide que possible après l'arrêt et compatible avec les règles d'exploitation. Ainsi, l'oxygénation, qui augmente l'activité de l'eau d'une manière considérable, se produit très rapidement et permet d'avancer la purification du fluide primaire. La décontamination du circuit est pratiquement nulle pendant la mise en arrêt à froid (3\% par jour). Cette procédure ne fait donc pas gagner directement de dose, mais elle a sa place dans cet historique, car elle permet de travailler plus rapidement à des ambiances relativement faibles.

\section{- Etat de surface des générateurs de vapeur (GV)}

L'état de surface des GV semble avoir une grande importance et l'on observe une corrélation assez nette entre les diverses fabrications et les débits de dose sur les boucles. En effet, les dépôts se font, pour certaines fabrications, préférentiellement dans les tubes. Les dépôts dans les boucles en sont d'autant plus faibles.

Des études sont en cours sur des traitements de surface particuliers, par exemple le polissage électrolytique. II semble que le traitement thermique ait une influence aussi pour des GV de fabrication comparable, mais ceci reste à vérifier.

\section{- Utilisation d'outillages spéciaux}

Des outillages spéciaux sont déjà utilisés (contrôles GV, bouchage tubes, MSDG (machine de serrage et desserrage des goujons, etc.) et d'autres sont à l'étude pour minimiser les doses prises par le personnel. Néanmoins, il faut rester à des niveaux de simplicité tels que la mise au point et la fiabilité des outillages permettent un gain réel en dose sur un bilan complet d'utilisation (installation, maintenance, efficacité, etc.).

\section{- Changement de nuance d'inconel des tubes GV}

Des études sont en cours concernant les relâchements relatifs en cobalt de l'inconel 600 (actuel) et du 690, mais de nombreux problèmes 
sont encore à résoudre. Néanmoins, les tubes des GV du palier N4 seront en inconel 690.

\section{- Grilles combustibles en zircaloy}

A terme, tous les assemblages comporteront des grilles en zircaloy, alliage qui est pratiquement neutre du point de vue de la corrosion. On attend une réduction de dose très importante (30\% sur les dépôts) du fait de la suppression des grilles inconel et donc du nickel et du cobalt sous flux.

Trois réacteurs ont déjà $1 / 3$ de cœur chargé avec des assemblages de ce type et des campagnes de mesure sont prévues pour les dépôts et les débits de dose sur différents circuits.

\section{- Organisation du travail}

Une approche systématique doit être conduite dans cette voie pour faire entrer les contraintes de radioprotection directement au niveau de la préparation des gammes d'intervention; de grands progrès ont déjà été réalisés. II reste, cependant, beaucoup de chemin à faire, car la radioprotection est actuellement ressentie comme un supplément de précautions à rajouter à celles déjà techniquement connues. II faut vraisemblablement aller plus loin et inclure dans la méthodologie de la préparation du travail l'idée même de radioprotection. Ceci ne concerne pas seulement les opérations les plus spectaculaires, car la dose totale est aussi la somme d'un grand nombre de très petites doses, dont certaines pourraient probablement être évitées.

D'autres actions de moindre envergure et de moindre incidence financière seront envisagées (baisse de la teneur en impuretés dans les matériaux, par exemple passage du débit RCV à $27 \mathrm{t} / \mathrm{h}$, etc.), mais qui doivent concourir à l'amélioration d'ensemble.

\section{LES PREVISIONS}

Des études statistiques fondées sur l'expérience ont permis d'établir une corrélation entre la dosimétrie globale et un indice dénommé indice d'activité. Cet indice est la moyenne arithmétique de 9 mesures de débits de dose au contact du circuit primaire, effectuées au moment où l'eau a une activité négligeable par rapport aux dépôts. La figure 10 récapitule les résultats pour l'ensemble des tranches de 900 MWe pour les différents cycles.

Par ailleurs, l'analyse et la modélisation de l'évolution dans le temps des divers produits de fission ont permis d'établir que l'état d'équilibre des tranches devrait se situer aux environs du dixième cycle, période à partir de laquelle la décroissance de l'activité des dépôts compense l'accroissement de la couche de dépôts.

Dans ces conditions, jusqu'à l'année dernière, la valeur avancée de l'indice à l'équilibre était basée particulièrement sur l'expérience de Tihange 
et Chooz, de l'ordre de $190 \mathrm{mrem} \cdot \mathrm{h}^{-1}$; elle conduit à une dose globale par tranche et par an de l'ordre de 450 h.rem.

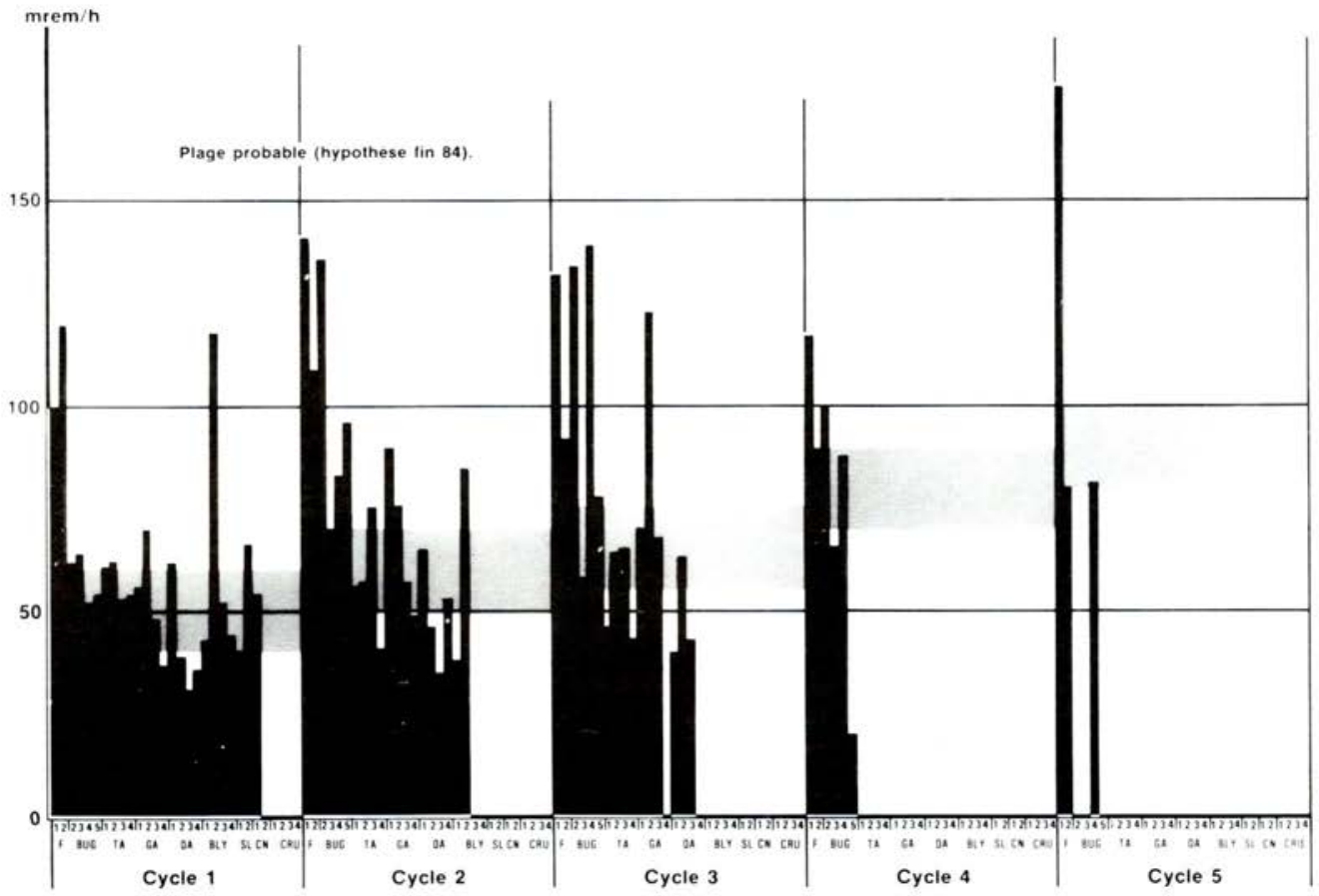

Fig. 10. - Débit de dose moyen sur les boucles primaires : indices d'activité.

Nous disposons aujourd'hui d'une expérience fondée sur plus de 80 arrêts de tranche, ce qui conduit à retenir une valeur asymptote de 130 mrem. $\mathrm{h}^{-1}$ (fig. 10). II faut signaler que ces estimations ont été effectuées à partir des valeurs relevées jusqu'en 1984, corrigées des doses dues aux modifications, celles-ci étant appelées à diminuer.

Sur ces bases, on estime ainsi qu'à l'équilibre, les doses pour visite normale devraient être de l'ordre de 210 h.rem pour une tranche et qu'une dose pour visite complète est de l'ordre de 410 h.rem. Dans ces conditions, la dose par réacteur et par an, définie comme la moyenne calculée sur 10 ans avec une seule visite complète et une visite normale par an, en tenant compte de la part hors arrêt, s'établira à 285 h.rem (fig. 1).

\section{CONCLUSION}

Cet exposé serait incomplet si l'on n'abordait pas la question du coût en exploitation de la radioprotection. Qu'il s'agisse des temps nécessaires 
à l'accueil et à la formation, du suivi médical ou du développement des films, des durées supplémentaires des opérations se déroulant en zone contrôlée, induites pour le conditionnement des locaux, du port de tenues particulières, une étude sommaire montre que, dans l'ensemble, il faut majorer les horaires d'environ $30 \%$, ce qui conduit, pour un arrêt moyen de 150000 heures environ, à un surcoût de l'ordre de 10 à $20 \%$ du coût de la révision.

Est-ce trop ? Ce n'est pas du tout certain, car investir dans la radioprotection, c'est aussi investir dans la fiabilité et la qualité. Notre intérêt commun, aux entreprises et à EdF, est bien de maintenir les centrales aussi propres que possible en améliorant l'organisation du travail. Ce dernier point devra être poursuivi par une analyse systématique des tâches faisant ressortir les populations les plus critiques et les opérations permettant d'économiser des doses, de manière à viser, dans chaque cas, l'efficacité économique en même temps que le nécessaire respect, et au-delà, des prescriptions de santé publique. 